Material performance

\title{
PHB-PEO electrospun fiber membranes containing chlorhexidine for drug delivery applications
}

\author{
J.G. Fernandes ${ }^{\mathrm{a}, \mathrm{b}}$, D.M. Correia ${ }^{\mathrm{a}, \mathrm{b}}$, G. Botelho ${ }^{\mathrm{a}}$, J. Padrão ${ }^{\mathrm{c}}$, F. Dourado ${ }^{\mathrm{c}}$, \\ C. Ribeiro ${ }^{\mathrm{b}}$, S. Lanceros-Méndez ${ }^{\mathrm{b}}$, V. Sencadas ${ }^{\mathrm{b}, \mathrm{d}, *}$ \\ ${ }^{a}$ Center/Department of Chemistry, University of Minho, Campus of Gualtar, 4710-057 Braga, Portugal \\ ${ }^{\mathrm{b}}$ Center/Department of Physics, University of Minho, Campus of Gualtar, 4710-057 Braga, Portugal \\ ${ }^{\mathrm{c}}$ Institute for Biotechnology and Bioengineering, Centre for Biological Engineering, University of Minho, Campus Gualtar, \\ 4710-057 Braga, Portugal \\ d School of Technology, Polytechnic Institute of Cávado and Ave, Campus of IPCA, 4750-810 Braga, Portugal
}

\section{A R T I C L E I N F O}

\section{Article history:}

Received 30 October 2013

Accepted 10 December 2013

\section{Keywords:}

Electrospun fibers

Drug release kinetics

Antibacterial activity

Biopolymers

Chlorhexidine

\begin{abstract}
A B S T R A C T
Fiber meshes of poly(hydroxybutyrate) (PHB) and poly(hydroxybutyrate)/poly(ethylene oxide) (PHB/PEO) with different concentrations of chlorhexidine (CHX) were prepared by electrospinning for assessment as a polymer based drug delivery system. The electrospun fibers were characterized at morphological, molecular and mechanical levels. The bactericidal potential of PHB and PHB/PEO electrospun fibers, with and without CHX, was investigated against Escherichia coli (E. coli) and Staphylococcus aureus (S. aureus) by disk diffusion susceptibility tests. Electrospun fibers containing CHX exhibited bactericidal activity. PHB/ PEO-1\%CHX displayed higher CHX release levels and equivalent antibacterial activity when compared to PHB/PEO with 5 and $10 \mathrm{wt} \% \mathrm{CHX}$. Bactericidal performance of samples with $1 \mathrm{wt} \% \mathrm{CHX}$ was assessed by Colony Forming Units (CFU), where reductions of $100 \%$ and 99.69\% against $E$. coli and $S$. aureus were achieved, respectively.
\end{abstract}

(c) 2013 Elsevier Ltd. All rights reserved.

\section{Introduction}

Material engineering approaches allow the design of materials with increasing complexity and functionality for application in the development of drug delivery systems [1]. Both natural and synthetic polymers are being used in controlled drug release to maximize system efficiency [1,2]. "Drug release" refers to the process in which drug solutes migrate from the initial position in the polymer system to the polymer outer surface and then to the medium [1]. This process is affected by multiple complex factors such as the structural characteristics of the material system, release environment and possible interactions between them [1].

\footnotetext{
* Corresponding author. School of Technology, Polytechnic Institute of Cávado and Ave, Campus of IPCA, 4750-810 Braga, Portugal.

E-mail addresses: vsencadas@fisica.uminho.pt, vsencadas@ipca.pt (V. Sencadas).
}

According to Zeng et al. [3], polymer based drug delivery systems show advantages when compared to the conventional dosage forms as they allow, for example, the therapeutic effect with reduced toxicity and enhance compliance of the patients by delivering drugs at a controlled rate over a period of time to the site of action [3]. However, some problems such as the low efficiency of the drug delivery systems and drug disintegration at the beginning of the process are still unresolved [3]. Drugs can be encapsulated directly into fibers processed by electrospinning, thus conferring on the electrospun fibers the function of drug carriers [3]. The study of electrospun fiber carriers for drug delivery is very limited [3]. Reported electrospun drug delivery systems include transdermal, oral sustained, targeted, implantable, tissue engineering and transmembrane delivery [4]. Drug delivery with polymer fibers is based on the principle that the delivery rate of the drug can be controlled by tailoring surface area of both drug and 
carrier [5]. Several factors that can affect the drug release from electrospun fibers are fiber geometry and thickness, membrane porosity, composition, crystallinity and swelling, to name a few [6].

Synthetic and natural biodegradable polymers have received special attention in pharmaceutical research [2]. Delivery systems based on biodegradable polymers are used because these polymers degrade into compounds that can be readily excreted from the body, thus obviating the need for later removal at the end of the treatment period [7].

Biodegradable polymers are used to control the drug release rate by diffusion through the polymer matrix or the pores within the matrix, and/or by degradation of the polymer chain and erosion of the matrix [2]. However, the control of the drug release rate from a delivery system that is susceptible to a degradation process is difficult, as the release rate may change over the degradation process [2].

PHB is a biocompatible polymer obtained from natural sources with a high degree of crystallinity. It is also characterized by high brittleness, poor processability and poor thermal stability [8]. Due to its natural origin, PHB has potential for biomedical applications, including drug delivery systems [9]. It has been evaluated for controlled drug release systems, surgical structures, wound dressings, orthopedic devices, tissue engineering and skin substitute materials [10].

Chlorhexidine (CHX) is one of the most efficient antimicrobial agents [11]. This drug has been widely used in a largely range of applications due to its antimicrobial activities against Gram-positive and Gram-negative bacteria and fungi, and non-toxicity toward mammalian cells $[11,12]$. It is used in several products for oral protection and, in general, for dentistry applications due to its antiseptic and disinfectant action on wounds [13]. Various studies $[11,12,14-16]$ have been conducted on CHX release, however, only a few works are devoted to $\mathrm{CHX}$ release with electrospun fibers. Chen et al. [12] studied electrospun cellulose acetate fibers containing $\mathrm{CHX}$ as a bactericide for Gram-negative Escherichia coli and the Gram-positive Staphylococcus epidermidis. It was concluded that $\mathrm{CHX}$ bound on cellulose acetate fibers is still capable of killing the bacteria with a reduction of $99.9 \%$ of the viable bacteria in 1 hour [12].

In an electrospinning process, a strong electrostatic field is applied to a polymer solution held in a syringe and feed through a needle [17]. The fiber jet travels through the atmosphere allowing the solvent to evaporate, thus leading to the deposition of solid polymer fibers on the collector [17].

In the present work PHB/PEO fiber membranes containing different amounts of CHX were produced by electrospinning. The influence of the presence of the drug on fiber diameter and average size distribution, as well as the evolution of the mechanical properties of the membranes was characterized. Further, drug immobilization on the fibers was confirmed and the $\mathrm{CHX}$ release kinetics evaluated. Bactericidal performance of PHB/PEO samples $1 \mathrm{wt} \% \mathrm{CHX}$ was assessed by Colony Forming Units (CFU), against both the gram-negative strain $E$. coli and gram-positive strain S. aureus.

\section{Experimental}

\subsection{Materials}

Poly(hydroxybutyrate), (PHB, molecular weight of $\sim 531112 \mathrm{Da}$ ) from sugar cane was supplied by PHB Industrial. Poly(ethylene oxide), (PEO, molecular weight of $\sim 100000 \mathrm{Da})$ was supplied by Polysciences and Chlorhexidine, (CHX, molecular weight of $\sim 505.45 \mathrm{Da}$ ) was purchased from Sigma Aldrich. The polymer solutions of PHB and PHB/PEO (90/10, wt\%) with different final CHX concentrations (1, 5 and $10 \mathrm{wt} \%$ ) were dissolved in a blend of N,N dimethylformamide (DMF, from Merck) and chloroform (CF, from Merck) $(80 / 20 \mathrm{v} / \mathrm{v})$ to achieve a final polymer concentration of $10 \%(\mathrm{w} / \mathrm{w})$. The polymer solutions with $\mathrm{CHX}$ were dissolved at $70{ }^{\circ} \mathrm{C}$ under stirring until complete dissolution.

\subsection{Electrospinning}

Electrospinning was performed by the method described previously [18]. Briefly, the polymer solution was placed in a glass syringe $(10 \mathrm{~mL})$ fitted with a steel needle with a diameter of $0.5 \mathrm{~mm}$. Electrospinning was conducted in a home-made controlled temperature chamber at $40{ }^{\circ} \mathrm{C}$, a relative humidity between $45-55 \%$ and applying a voltage of $20 \mathrm{kV}$ with a power supply from Glassman (model PS/ FC30P04). A syringe pump (from Syringepump) was used to feed the polymer solutions into the needle tip at a rate of $10 \mathrm{~mL} . \mathrm{h}^{-1}$. The as-spun randomly oriented fibers were collected on a grounded collecting plate and stored at room temperature.

\subsection{Electrospun fiber membrane characterization}

Electrospun fiber membranes were coated with a thin gold layer using a sputter coater (Polaron, model SC502), and their morphology was analyzed using scanning electron microscopy (SEM) (Quanta 650 from FEI) with an accelerating voltage of $10 \mathrm{kV}$. The fiber average diameter and their size distribution was calculated over approximately 40 fibers using SEM images at 5000X magnification and Image J software. Contact angle measurements (sessile drop in dynamic mode) were performed at room temperature in a Data Physics OCA20 device using ultrapure water as test liquid. The contact angles were measured by depositing water drops $(3 \mu \mathrm{L})$ on the sample surface and analyzed with SCA20 software. At least 6 measurements on each sample were performed in different membrane locations, and the average contact angle was taken as the result for each sample.

Infrared measurements (FTIR) were performed at room temperature with an ABB FTLA 2000 apparatus in transmission mode from 4000 to $500 \mathrm{~cm}^{-1}$. FTIR spectra were collected after 10 scans with a resolution of $4 \mathrm{~cm}^{-1}$. The measurements were performed with dry potassium bromide pellets $(\mathrm{KBr})$.

The mechanical properties of the electrospun fiber membranes (dimensions of $40 \mathrm{~mm} \times 5 \mathrm{~mm} \times 40 \mu \mathrm{m}$ ) were characterized by stress-strain experiments in tensile mode with a Linkam TST 350 universal testing machine. The 
tensile experiments were carried out at a deformation rate of $0.5 \mathrm{~mm} \cdot \mathrm{min}^{-1}$ at room temperature. Elastic modulus $(E)$ was obtained through Hook's law, relating the stress $(\sigma)$ and the strain $(\varepsilon)$ for the uniaxial deformation [19]:

$\sigma=E \varepsilon$

Mechanical results are reported as averages of the data taken from at least five specimens.

\section{4. "In vitro" drug release study}

The electrospun fiber membranes of PHB and PHB/PEO with different $\mathrm{CHX}$ concentrations were dried, cut into $13 \mathrm{~mm}$ diameter pieces (triplicate samples were used for statistical purposes), and the initial weight was measured. Each specimen was then placed in a tube containing $10 \mathrm{~mL}$ of phosphate-buffered saline solution (PBS, pH 7.4). The tubes containing the solutions were then incubated in an air circulation oven (HERAEUS Vacuotherm) at $37{ }^{\circ} \mathrm{C}$ for 72 days. The PBS solution was removed from the tubes and renewed every three days for the samples with higher incubation times; for shorter times, the solution was removed and measured.

The amount of $\mathrm{CHX}$ released from the nanofibers to the PBS solution was measured using a UV-Vis spectrophotometer (UV - 2501 PC Shimadzu) by measuring the absorbance at $254 \mathrm{~nm}$ [12] using a $1 \mathrm{~cm}$ quartz cell. A 50 ppm stock solution of CHX in PBS was used to prepare standard solutions with different concentrations (from 0 up to $-10 \mathrm{ppm}$ ) in order to obtain the calibration curve.

\subsection{Antibacterial activity}

\subsubsection{Disk diffusion susceptibility test}

In order to determine materials' antibacterial properties, $5 \mathrm{~mm}$ diameter sample discs were submitted to a disk diffusion experimental procedure based on the procedure described in [20]. Escherichia coli and Staphylococcus aureus bacteria pre-inocula (kindly provided by the Faculty of Pharmacy, University of Porto, Portugal) were incubated in Mueller Hinton Agar (Himedia) culture medium overnight at $37{ }^{\circ} \mathrm{C}$. The discs were sterilized using ultraviolet (UV) light. McFarland 0.5 turbidity standard was used as a reference to inoculate fresh Mueller Hinton Agar plates with 1-2 $\times 10^{8}$ CFUs (Colony Forming Units) $\mathrm{mL}^{-1}$ of each bacteria in PBS, $\mathrm{pH} 7.4$, that were evenly spread on the medium surface. The membrane samples were placed onto the inoculated plates and were incubated for $24 \mathrm{~h}$ at $37{ }^{\circ} \mathrm{C}$. High resolution pictures were acquired and the zone of inhibition (ZoI) was calculated using Image J software. The statistical analysis of One-way ANOVA and Tukey post-tests were performed using GraphPad Prism 4 software.

\subsubsection{Evaluation of contact bactericidal effectiveness}

The evaluation of the contact antibacterial efficiency of the PHB/PEO and PHB/PEO with 1 wt\% CHX fiber membranes was performed according to the AATCC Test Method 100-2012 [21]. The pre-inocula of the bacteria were incubated overnight in Nutrient Broth (Himedia).
The sample patches, previously sterilized by UV light, were inoculated with $2.5 \times 10^{6}$ CFUs $\mathrm{mL}^{-1}$ of $E$. coli and $2.5 \times 10^{5}$ CFUs $\mathrm{mL}^{-1}$ S. aureus diluted in PBS, $\mathrm{pH}$ 7.4. To determine the percentage of $\mathrm{CFU}$ reduction the following formula was used:

$R=\frac{\mathrm{A}-\mathrm{B}}{\mathrm{A}} \times 100$

where $R$ corresponds to the percentage reduction, $A$ to the colony count obtained immediately after the inoculation of the samples and $B$ to the bacteria count after $24 \mathrm{~h}$ of incubation at $37^{\circ} \mathrm{C}$. All colonies were counted visually.

\section{Results and discussion}

\subsection{Fiber membrane morphology}

Solutions of $\mathrm{PHB} / \mathrm{CHX}$ were impossible to electrospin into smooth and bead free fibers, even for small amounts of drug. A blend of PHB/PEO (90/10, w/w) was prepared and the electrospinning process could be performed. Moreover, different amounts of $\mathrm{CHX}$ (1, 5 and $10 \mathrm{wt} \%)$ were added to the solution and suitably electrospun. PEO was chosen as a blending material to stabilize the electrospinning process of PHB/PEO with CHX, as it has been demonstrated that the addition of a small amount of PEO into the solution facilitates the electrospinning process by increasing the solution elasticity [12]. PEO is also miscible with PHB due to the specific interactions of the PHB carbonyl groups with the $\mathrm{CH}_{2}$ groups of PEO [22,23].

Further, CF does not allow CHX dissolution and the presence of drug clusters in the solution was visually observed, even after $2 \mathrm{~h}$ in a water bath with ultrasound. It was reported that DMF is a good CHX solvent and, additionally, due to the low dielectric constant and boiling point [18], the addition of a small amount of DMF to the solution mixture allows $\mathrm{CHX}$ dissolution, promoting better distribution of the drug among the fibers and helps to stabilize the electrospinning process.

As-spun PHB and PHB/PEO membranes without CHX showed a smooth surface, without beads, and a random fiber distribution (Fig. 1). Further, PHB/PEO electrospun samples with 1 and 5 wt\% CHX showed a similar morphology to that found for the pristine electrospun samples but, on the other hand, fibers with $10 \mathrm{wt} \% \mathrm{CHX}$ are thinner and show higher amounts of beads (Fig. 1d).

The mean diameter of $\mathrm{PHB} / \mathrm{PEO}$ fibers range from $1.5 \pm 0.4 \mu \mathrm{m}$ up to $1.2 \pm 0.9 \mu \mathrm{m}$, and is influenced by the presence of the CHX in the solution (Fig. 2). It was observed that the incorporation of drug in the polymeric solution leads to a broader fiber size distribution, especially for small amounts of CHX (Fig. 2).

The applied voltage is the drive of the electrospinning process, and fiber formation is mainly achieved by the stretching and acceleration of the jets promoted by the high electrical field. When a high electric field is applied between the needle tip and a grounded collector, formation of a higher charge density on the surface of the ejected jets occurs; thus the jet velocity increases and higher elongation forces are imposed on the jet $[24,25]$. CHX has two 


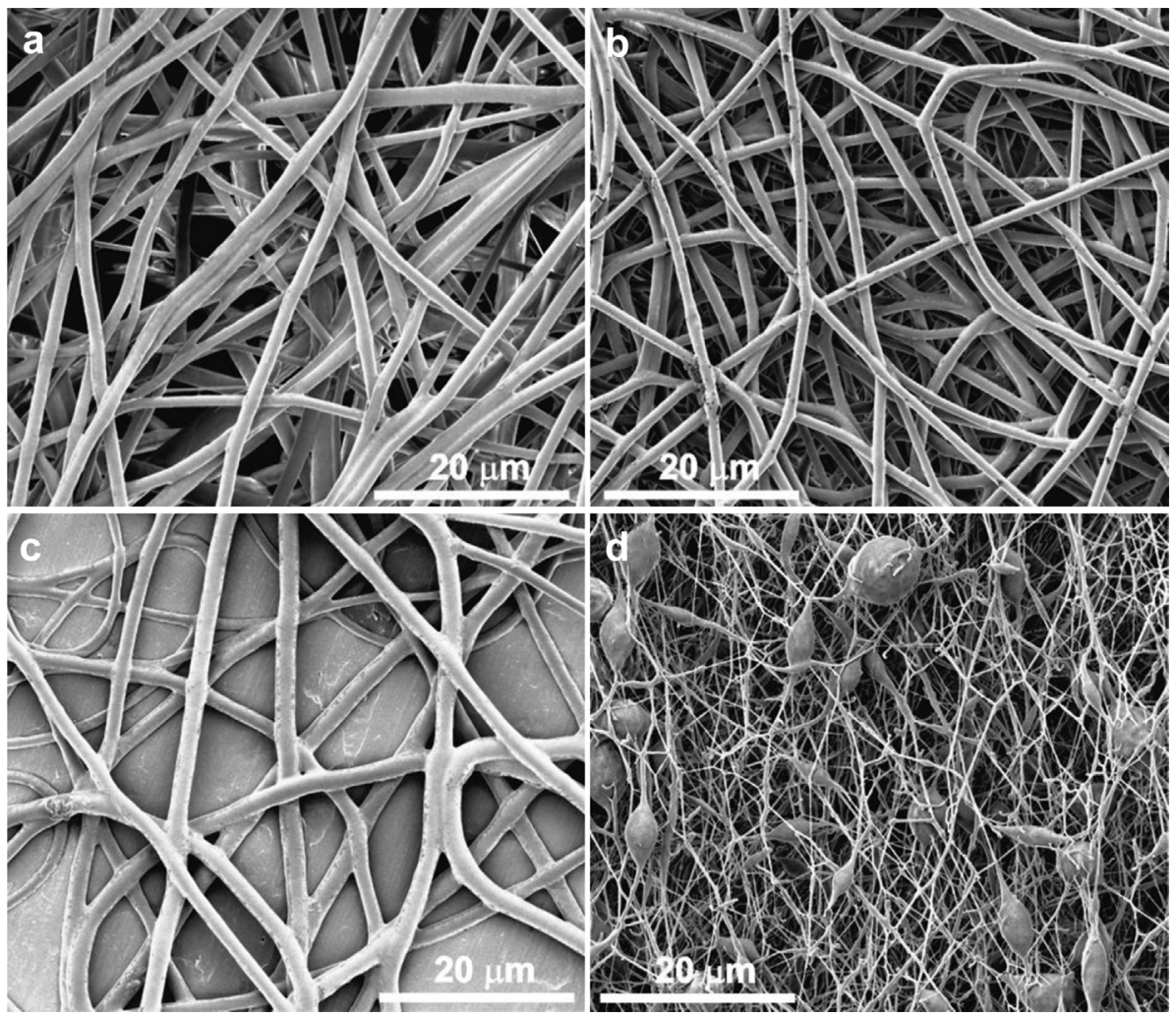

Fig. 1. Morphology of the electrospun fiber membranes of (a) PHB, (b) PHB/PEO, and PHB/PEO with 1 wt\% (c) and 10 wt\% (d) of CHX concentration.
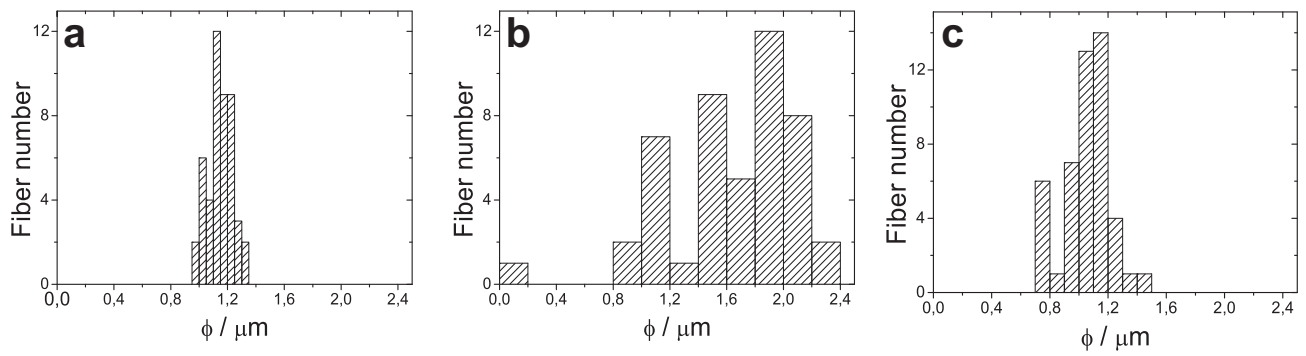

Fig. 2. PHB/PEO fiber mean diameter with different $\mathrm{CHX}$ concentrations.

cationic groups separated by a hydrophobic bridging structure (hexamethylene) and, when a small amount of drug is added to the solution, the charges carried by the solution increase, promoting higher stretching of the jet, leading to the formation of smooth and thinner fibers. However, when CHX is above a certain threshold (possibly between 5 and $10 \mathrm{wt} \%$ ), the charges present in the solution lead to higher jet stretch ratios at a given electric field, leading to the jet disintegration and the formation of beads (Fig. 1) [24].

Fiber membrane wettability was qualitatively evaluated by water contact angle measurements. PHB has hydrophobic behavior with a water contact angle of $126 \pm 3^{\circ}$, while for the samples containing PEO with and without CHX the water was absorbed almost immediately by the fiber membrane, which is related to the hydrophilic nature of PEO and CHX (data no shown) [3,12,26].

\subsection{Fourier transformed infrared spectroscopy}

In order to characterize the interaction between the polymer blend and the CHX, Fourier transform infrared spectroscopy (FTIR) was performed (Fig. 3). The FTIR spectra show the characteristic vibrational bands of $\mathrm{CHX}$ at $1665 \mathrm{~cm}^{-1}$ corresponding to the $\mathrm{C}=\mathrm{N}-\mathrm{H}$ stretching vibration [27], as well as the absorption bands corresponding to the aromatic $\mathrm{C}=\mathrm{C}$ stretching located at 1537 and $1490 \mathrm{~cm}^{-1}$ [12]. In this way, the presence of the CHX in the membranes after processing was confirmed [28].

\subsection{Mechanical properties}

Mechanical integrity of polymer membrane is an important practical aspect, because material often requires manipulation and sample integrity must be maintained. It 


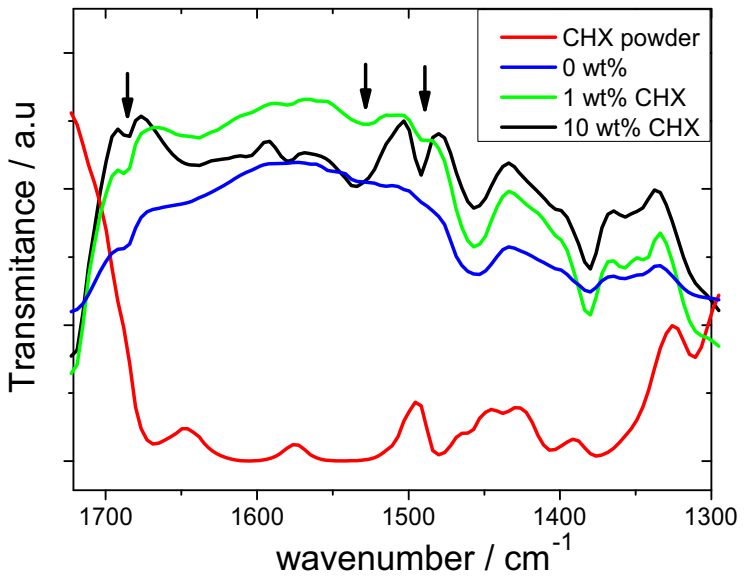

Fig. 3. FTIR spectra of $\mathrm{CHX}$ and $\mathrm{PHB} / \mathrm{PEO}$ electrospun membranes with 0,1 and $10 \mathrm{wt} \%$ of incorporated drug.

was experimentally observed that the samples with higher amounts of drug were very fragile and they easily broke during clamping. Further, the influence of PEO and CHX on the overall mechanical properties of random fiber membranes was evaluated through stress-strain measurements (Fig. 4).

The incorporation of PEO in the PHB blend promotes an increase of the deformation at break (Fig. 4) without compromising the elastic modulus when compared to the PHB polymer. This effect is promoted by blending a hard (PHB) with a soft component (PEO). Further, when CHX is added to the $\mathrm{PHB} / \mathrm{PEO}$ polymer blend, the deformation at break is reduced but the elastic modulus increases (Table 1). PHB films are known to have fragile behavior with a deformation at break below $5 \%$ and an elastic modulus around $1.5 \mathrm{GPa}[29,30]$. The brittleness of PHB was attributed to polymer glass transition that occurs $\sim 0{ }^{\circ} \mathrm{C}$, leading to cold recrystallization at room temperature, which restricts the mobility of the amorphous phase in interfibrillar and/or interlamellar regions, and results in a failure at relatively low strains [31].

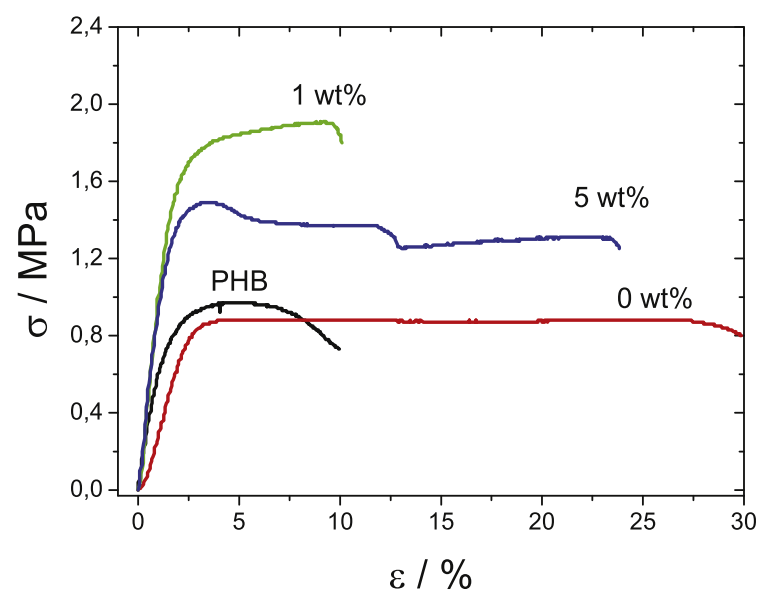

Fig. 4. Stress-strain curves for PHB and PHB/PEO with 0,1 and 5 wt\% of CHX.
Table 1

Mechanical properties of PHB and PHB/PEO with different CHX concentrations (all values are expressed as mean values \pm standard deviation (SD)).

\begin{tabular}{llll}
\hline Sample & $\mathrm{E}(\mathrm{MPa})$ & $\sigma_{\max }(\mathrm{MPa})$ & $\varepsilon_{\text {break }}(\%)$ \\
\hline PHB & $54 \pm 5$ & $0.95 \pm 0.3$ & $10 \pm 3$ \\
$0 \mathrm{wt} \%$ & $38 \pm 15$ & $0.87 \pm 0.2$ & $30 \pm 6$ \\
$1 \mathrm{wt} \%$ & $93 \pm 15$ & $1.91 \pm 0.4$ & $10 \pm 3$ \\
$5 \mathrm{wt} \%$ & $97 \pm 9$ & $1.49 \pm 0.3$ & $23 \pm 4$ \\
\hline
\end{tabular}

When CHX is added to the polymer fibers, an increase of elastic modulus accompanied by a decrease of the strain at break was observed when $\mathrm{CHX}$, probably due to the strong interfacial interactions between the matrix and the filler. Mechanical properties of fibrous membranes are deeply influenced by the combination of several factors such polymer intrinsic properties, fiber diameter and packing density, and even porosity [32]. Bianco et al. [29] reported that elastic modulus, maximum stress and strain at break of poly(3-hydroxybutyrate-co-3-hydroxyvalerate) (PHBV) blended with PEO decreased with increase of the PEO content, and they attributed this effect to the formation of co-continuous fibers made up of the two polymeric components, with consequent combination of their mechanical properties.

\subsection{CHX release kinetics}

Drug release from the polymer fiber membranes is higher for the samples with lower drug contents incorporated into the polymer blend. In the case of electrospun cellulose acetate fibers, the bound concentration of $\mathrm{CHX}$ to electrospun cellulose acetate fibers varied in a narrow range between 5 and $9 \mathrm{wt} \%$, this being the effect of $\mathrm{CHX}$ on binding and drug release weak for concentrations below 2 wt\% [12]. Similarly, the present results indicate that the amount of unbound CHX present in the PHB/PEO electrospun membranes is larger for the samples with $1 \mathrm{wt} \%$ of $\mathrm{CHX}$, when compared to the samples with higher amounts of $\mathrm{CHX}$. The sample with $5 \mathrm{wt} \% \mathrm{CHX}$ should be more bound to the polymer structure and, consequently, lower drug contents are released to the PBS solution (Fig. 5).

Various empiric kinetics models described the overall release of drug from dosage forms [33]. The KorsmeyerPeppas (KP) model describes the phenomena of drug release from a polymeric system:

$\frac{M_{t}}{M_{\infty}}=K t^{n}$

where $M_{t} / M_{\infty}$ is defined as the fraction of drug released at time $t, K$ is the release rate constant and $n$ is the release exponent and is used to characterize the different release mechanisms [33]. The fitting results to the experimental data are presented in Fig. 5 and Table 2.

In the KP model, $n$ is indicative of the mechanism of drug diffusional release that occurs by $\mathrm{CHX}$ molecular diffusion. According to Korsmeyer et al. [34], if $0.5 \leq n$, the drug release is ruled by a anomalous diffusion mechanism. It was observed that the $n$ parameter obtained for the $\mathrm{CHX}$ release fitting results decreases from 0.21 to 0.15 with 


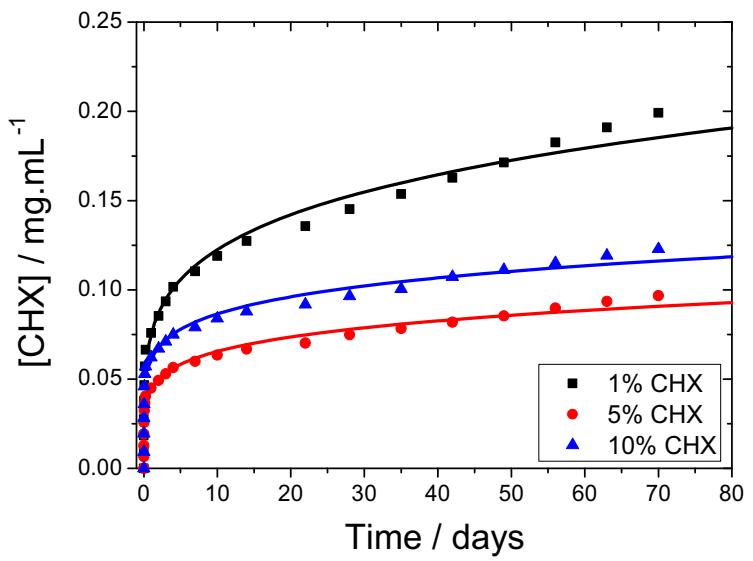

Fig. 5. Drug release curves of electrospun fibers with CHX. Dots corresponds to experimental data and the solid lines to the fitting results.

Table 2

Drug release kinetics parameters obtained by the Korsmeyer-Peppas model.

\begin{tabular}{llll}
\hline CHX (wt\%) & $\mathrm{K}\left(\right.$ days $\left.^{-\mathrm{n}}\right)$ & $\mathrm{n}$ & $\mathrm{R}^{2}$ \\
\hline 1 & $75.2^{*} 10^{-3}$ & 0.21 & 0.98 \\
5 & $44.5^{*} 10^{-3}$ & 0.17 & 0.98 \\
10 & $61.1^{*} 10^{-3}$ & 0.15 & 0.97 \\
\hline
\end{tabular}
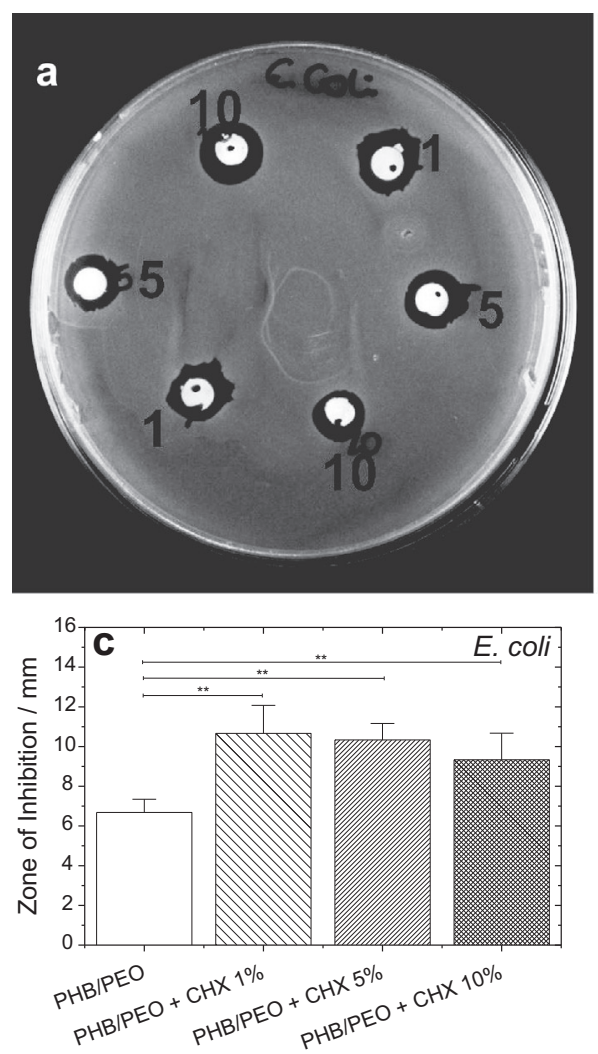

increasing of the drug content present in the polymer fibers mats (Table 2), which suggests that CHX molecules were distributed along the polymer fibers, promoting rapid diffusion out at the initial release time [4].

\subsection{Antibacterial activity}

The bacteria E. coli and S. aureus were used as indicators for antibacterial efficiency of the polymer system containing CHX. The bactericidal capacity of the different concentrations of $\mathrm{CHX}$ was determined using disk diffusion tests. The contact killing efficiency of PHB/PEO with different $\mathrm{CHX}$ contents was assessed by $\mathrm{CFU}$ quantification (Fig. 6). All the CHX concentrations exhibited significant bactericidal properties when compared to the experiment control of PHB/PEO fibers without CHX (Fig. 6c and d, E. coli $P<0.01 ;$ S. aureus $P<0.001$ ), reflecting the effective antibacterial activity for this polymeric system. Moreover, for each microorganism, the zones of inhibition generated for the tested concentrations of CHX presented no significant differences among them, which demonstrates that the PHB/PEO fiber mats with 1, 5 and $10 \mathrm{wt} \%$ of CHX possess equivalent bactericidal potency. The antibacterial efficiency was higher against $S$. aureus in comparison to E. coli. For instance the PHB/PEO $1 \mathrm{wt} \%$ CHX samples presented a ZoI in $S$. aureus roughly two fold higher than in gram-negative $E$. coli. This was expected as gram-positive S. aureus bacteria
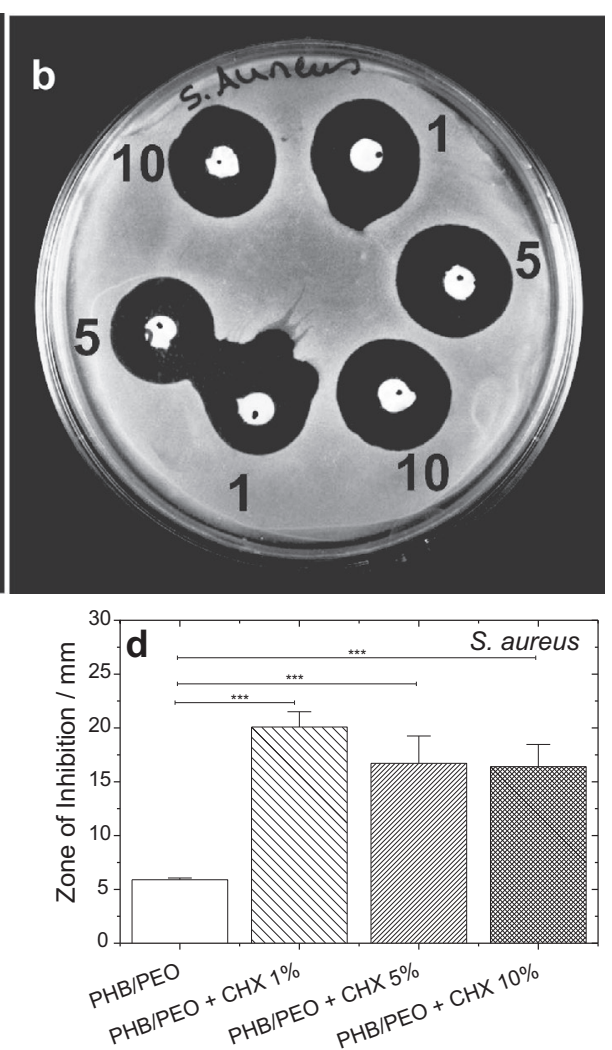

Fig. 6. PHB/PEO with 1, 5 and 10 wt\% CHX antibacterial activity assays against E. Coli (a) and S. aureus (b). Zone of inhibition (ZoI) obtained for PHB/PEO control and PHB/PEO with 1,5 and $10 \mathrm{wt} \% \mathrm{CHX}$ against $E$. Coli (c) and S. aureus (d). One-way ANOVA with Tukey multiple comparison test, ${ }^{* \star}$ corresponds to $P<0.01$ and *** corresponds to $P<0.001$. 
Table 3

Colony forming unit (CFU) count immediately after inoculation ( $0 \mathrm{~h})$ and after $24 \mathrm{~h}$ of incubation. Bacteria reduction was calculated using Equation 2.

\begin{tabular}{|c|c|c|c|c|}
\hline & & \multicolumn{2}{|c|}{ CFU's count (CFU mL $\left.{ }^{-1}\right)$} & \multirow{2}{*}{$\begin{array}{l}\text { Bacteria } \\
\text { reduction } \\
(\%)\end{array}$} \\
\hline & & Time $(0 \mathrm{~h})$ & Time $(24 \mathrm{~h})$ & \\
\hline$\overline{\mathrm{PHB} / \mathrm{PEO}}$ & $\begin{array}{l}\text { Escherichia coli } \\
\text { Staphylococcus } \\
\text { aureus }\end{array}$ & $\begin{array}{l}1.44 \times 10^{6} \\
1.70 \times 10^{5}\end{array}$ & $\begin{array}{l}3.93 \times 10^{7} \\
7.94 \times 10^{4}\end{array}$ & $\begin{array}{l}-2.69 \times 10^{3} \\
59.26\end{array}$ \\
\hline $\begin{array}{l}\mathrm{PHB} / \mathrm{PEO} \\
1 \% \mathrm{CHX}\end{array}$ & $\begin{array}{l}\text { Escherichia coli } \\
\text { Staphylococcus } \\
\text { aureus }\end{array}$ & $\begin{array}{l}0 \\
3.19 \times 10^{3}\end{array}$ & $\begin{array}{l}0 \\
10\end{array}$ & $\begin{array}{l}100 \\
99.68\end{array}$ \\
\hline
\end{tabular}

are more susceptible to the biocidal action of this biguanide than E. coli [35-37]; indeed, the value of CHX minimal inhibitory concentration (MIC) is approximately a half for $S$. aureus $\left(0.5-4 \mu \mathrm{g} \mathrm{mL}^{-1}\right)$ that for E. coli $\left(2-8 \mu \mathrm{g} \mathrm{mL}^{-1}\right)[12,35]$.

The sample with $1 \mathrm{wt} \% \mathrm{CHX}$ was chosen to quantify the ability of the composite samples to eliminate the tested bacteria ( $E$. coli and S. aureus), because of its higher release of the drug to the aqueous media (Fig. 5). Table 3 displays the results obtained in the contact-killing experiment. As can be observed, electrospun fibers without $\mathrm{CHX}$ did not inhibit $E$. coli growth on contact, and an increase in bacteria number was observed. However, PHB/PEO showed a considerable reduction in $S$. aureus viable cell count after $24 \mathrm{~h}$ of incubation (Table 3 ).

It was observed that $\mathrm{PHB} / \mathrm{PEO}$ with $1 \% \mathrm{CHX}$ produced a steep reduction of the viable bacterial cells in a very short time. This was particularly so on $E$. coli, since the CFU count immediately after inoculation was 0 , representing complete kill $(>6-\log )$. As for $S$. aureus, for $0 \mathrm{~h}$ the CFU count would be similar in both electrospun materials, but a 2-log reduction was identified for the sample with $1 \mathrm{wt} \% \mathrm{CHX}$. Such a rapid bactericidal action is only possible due to the mechanism of action of $\mathrm{CHX}$. This biguanide has a cationic nature which electrostatically attracts it to the negatively charged bacteria allowing a contact-killing capacity of $\log 4$ (99.99\%) in a wide scope of bacteria in just 10 minutes at a concentration of $0.02 \%$ [38,39]. Taking into consideration that the electrospun material produced possesses $1 \mathrm{wt} \%$ of $\mathrm{CHX}$, a highly effective bactericidal action is expected even if exposure occurs for a very short period of time. However, $S$. aureus displayed a higher susceptibility in disc diffusion tests, showing a resistance in this contact-killing assay. Several factors may be responsible for this unforeseen survival rate that required further testing to pin-point the reason. There is a possibility that a portion of the $S$. aureus suspension may have been exposed to a sub lethal dosage of CHX and only suffered a bacteriostatic effect [38]. A reduction of $99.6 \%$ was observed for the $\mathrm{PHB} / \mathrm{PEO}$ with $1 \mathrm{wt}$ \% $\mathrm{CHX}$, which represents a 2-log reduction.

\section{Conclusions}

Bactericide fiber membranes were produced by electrospinning of polymer blends with different amounts of chlorhexidine for assessment as a polymer based drug delivery system. The addition of a small amount of a high molecular weight PEO to the PHB solutions significantly improves the elasticity of the PHB solution and facilitates the formation of fibers. Further, it was observed that for CHX concentrations above 5\% present in the polymer solution the electrospinning process was unstable due to the charges added by the drug to the solution that promotes higher stretch ratios of the jet, leading to the formation of fibers and beads due to poor polymer entanglement.

An increase of polymer membrane elastic modulus was observed for the samples with $\mathrm{CHX}$ incorporated and the deformation at break decreases when compared to the $\mathrm{PHB} / \mathrm{PEO}$ fiber membranes without CHX. Drug release in a PBS solution at $37{ }^{\circ} \mathrm{C}$ was characterized and it was found that the sample with less CHX present in the polymeric solution delivered higher amounts of the drug to the buffer solution. CHX release kinetics was studied and KP model shows that the drug release mechanism is ruled by an anomalous diffusion mechanism.

The bactericidal potential of PHB and PHB/PEO electrospun fibers with and without $\mathrm{CHX}$ was investigated against Escherichia coli (E. coli) and Staphylococcus aureus (S. aureus) by disk diffusion susceptibility tests. Bactericidal performance of samples with $1 \mathrm{wt} \% \mathrm{CHX}$ presents a reduction of $100 \%$ and $99.69 \%$ against $E$. coli and $S$. aureus, respectively. Finally, the strategy present in this work is not limited to the incorporation of the $\mathrm{CHX}$, but will also enable incorporation of a wide range of pharmaceutical drugs into the fibers.

\section{Acknowledgments}

This work was supported by FEDER through the COMPETE Program and by the Portuguese Foundation for Science and Technology (FCT) in the framework of the Strategic Project PEST-C/FIS/UI607/2011 and PEST-C/QUI/ UIO686/2011. The authors also thank funding from Matepro - Optimizing Materials and Processes", ref. NORTE-070124-FEDER-000037", co-funded by the "Programa Operacional Regional do Norte" (ON.2 - O Novo Norte), under the "Quadro de Referência Estratégico Nacional" (QREN), through the "Fundo Europeu de Desenvolvimento Regional" (FEDER). D.M.C, JP and VS thanks the FCT for the, SFRH/BD/82411/2011, SFRH/BD/64901/2009 and SFRH/ BPD/63148/2009 grants respectively. The authors also thank support from the COST Action MP1003, 2010 'European Scientific Network for Artificial Muscles' and to the COST Action MP1206 'Electrospun Nano-fibres for Bio inspired Composite Materials and Innovative Industrial Applications'. The authors also thank prof. José Luis Gomez Ribelles from the Unversidad Politécnica de Valencia, Spain, for interesting discussions on these issues.

\section{References}

[1] K.W. Fu Y, Drug Release Kinetics and Transport Mechanisms of Nondegradable and Degradable Polymeric Delivery Systems, vol. 7, 2010, pp. 429-444.

[2] L. Tuovinen, S. Peltonen, K. Järvinen, Drug release from starchacetate films, Journal of Controlled Release 91 (2003) 345-354.

[3] J. Zeng, X. Xu, X. Chen, Q. Liang, X. Bian, L. Yang, X. Jing, Biodegradable electrospun fibers for drug delivery, Journal of Controlled Release 92 (2003) 227-231.

[4] K.N. Kontogiannopoulos, A.N. Assimopoulou, I. Tsivintzelis, C. Panayiotou, V.P. Papageorgiou, Electrospun fiber mats containing 
shikonin and derivatives with potential biomedical applications, International Journal of Pharmaceutics 409 (2011) 216-228.

[5] H. Peng, S. Zhou, T. Guo, Y. Li, X. Li, J. Wang, J. Weng, In vitro degradation and release profiles for electrospun polymeric fibers containing paracetanol, Colloids and Surfaces B: Biointerfaces 66 (2008) 206-212.

[6] M.V. Natu, H.C. de Sousa, M.H. Gil, Effects of drug solubility, state and loading on controlled release in bicomponent electrospun fibers, International Journal of Pharmaceutics 397 (2010) 50-58.

[7] J.S. Im, J. Yun, Y.-M. Lim, H.-I. Kim, Y.-S. Lee, Fluorination of electrospun hydrogel fibers for a controlled release drug delivery system, Acta Biomaterialia 6 (2010) 102-109.

[8] J.W. You, H.J. Chiu, T.M. Don, Spherulitic morphology and crystallization kinetics of melt-miscible blends of poly(3-hydroxybutyrate) with low molecular weight poly(ethylene oxide), Polymer 44 (2003) 4355-4362.

[9] A.Q. Ali, T.P. Kannan, A. Ahmad, A.R. Samsudin, In vitro genotoxicity tests for polyhydroxybutyrate - a synthetic biomaterial, Toxicology in Vitro 22 (2008) 57-67.

[10] A.S. Asran, K. Razghandi, N. Aggarwal, G.H. Michler, T. Groth, Nanofibers from blends of polyvinyl alcohol and polyhydroxy butyrate as potential scaffold material for tissue engineering of skin, Biomacromolecules 11 (2010) 3413-3421.

[11] E. Verraedt, M. Pendela, E. Adams, J. Hoogmartens, J.A. Martens, Controlled release of chlorhexidine from amorphous microporous silica, Journal of Controlled Release 142 (2010) 47-52.

[12] L. Chen, L. Bromberg, T.A. Hatton, G.C. Rutledge, Electrospun cellulose acetate fibers containing chlorhexidine as a bactericide, Polymer 49 (2008) 1266-1275.

[13] R. Scaffaro, L. Botta, M. Sanfilippo, G. Gallo, G. Palazzolo, A.M. Puglia, Combining in the melt physical and biological properties of poly(caprolactone) and chlorhexidine to obtain antimicrobial surgical monofilaments, Applied Microbiology and Biotechnology 97 (2013) 99-109.

[14] N. Meng, N.-L. Zhou, S.-Q. Zhang, J. Shen, Controlled release and antibacterial activity chlorhexidine acetate (CA) intercalated in montmorillonite, International Journal of Pharmaceutics 382 (2009) $45-49$.

[15] N.J. Medlicott, I.G. Tucker, M.J. Rathbone, D.W. Holborow, D.S. Jones, Chlorhexidine release from poly( $\varepsilon$-caprolactone) films prepared by solvent evaporation, International Journal of Pharmaceutics 143 (1996) 25-35.

[16] E. Farkas, D. Kiss, R. Zelkó, Study on the release of chlorhexidine base and salts from different liquid crystalline structures, International Journal of Pharmaceutics 340 (2007) 71-75.

[17] V. Sencadas, D.M. Correia, C. Ribeiro, S. Moreira, G. Botelho, J.L. Gómez Ribelles, S. Lanceros-Mendez, Physical-chemical properties of cross-linked chitosan electrospun fiber mats, Polymer Testing 31 (2012) 1062-1069.

[18] D.M. Correia, C. Ribeiro, J.C.C. Ferreira, G. Botelho, J.L.G. Ribelles, S. Lanceros-Méndez, V. Sencadas, Influence of electrospinning parameters on poly(hydroxybutyrate) electrospun membranes fiber size and distribution, Polymer Engineering \& Science (2013), http:// dx.doi.org/10.1002/pen.23704.

[19] K.P. Menard, Dynamic Mechanical Analysis: A Practical Introduction, Taylor \& Francis, 2002

[20] I. Wiegand, K. Hilpert, R.E.W. Hancock, Agar and broth dilution methods to determine the minimal inhibitory concentration (MIC) of antimicrobial substances, Nature Protocols 3 (2008) 163-175.

[21] A.A.o.T.C.a. Colorists, Antibacterial Finishes on Textile Materials: Assessment of in: Test Method 100-TM100, America Association of Textile Chemists and Colorists, North Carolina, 2012.
[22] H.J. Choi, S.H. Park, J.S. Yoon, H.S. Lee, S.J. Choi, Rheological study on poly-D(-)(3-hydroxybutyrate) and its blends with poly(ethylene oxide), Polymer Engineering \& Science 35 (1995) 1636-1642.

[23] H. Yang, L. Ze-Sheng, H.-j. Qian, Y.-b. Yang, X.-b. Zhang, C.-c. Sun, Molecular dynamics simulation studies of binary blend miscibility of poly(3-hydroxybutyrate) and poly(ethylene oxide), Polymer 45 (2004) 453-457.

[24] S. Ramakrishna, An Introduction to Electrospinning and Nanofibers, World Scientific Publishing Company Incorporated, 2005.

[25] V. Sencadas, C. Ribeiro, J. Nunes-Pereira, V. Correia, S. LancerosMéndez, Fiber average size and distribution dependence on the electrospinning parameters of poly(vinylidene fluoride-trifluoroethylene) membranes for biomedical applications, Applied Physics A 109 (2012) 685-691.

[26] M. Amiji, K. Park, Surface modification of polymeric biomaterials with poly(ethylene oxide), in: Polymers of Biological and Biomedical Significance, American Chemical Society, 1993, pp. 135-146.

[27] W. Musial, The deposition of chlorhexidine on chemically modified thermosensitive polyNIPA microgels assessed by EDXS in scanning electron microscopy, Latin American Journal of Pharmacy 30 (2011) $1481-1486$.

[28] N. Fong, L.A. Poole-Warren, A. Simmons, Development of sustainedrelease antibacterial urinary biomaterials through using an antimicrobial as an organic modifier in polyurethane nanocomposites, Journal of Biomedical Materials Research Part B: Applied Biomaterials 101B (2013) 310-319.

[29] A. Bianco, M. Calderone, I. Cacciotti, Electrospun PHBV/PEO cosolution blends: microstructure, thermal and mechanical properties, Materials Science and Engineering: C 33 (2013) 1067-1077.

[30] O. Suwantong, S. Waleetorncheepsawat, N. Sanchavanakit, P. Pavasant, P. Cheepsunthorn, T. Bunaprasert, P. Supaphol, In vitro biocompatibility of electrospun poly(3-hydroxybutyrate) and poly(3-hydroxybutyrate-co-3-hydroxyvalerate) fiber mats, International Journal of Biological Macromolecules 40 (2007) 217-223.

[31] S.H. El-Taweel, B. Stoll, G.W.H. Höhne, A.A. Mansour, H. Seliger, Stress-strain behavior of blends of bacterial polyhydroxybutyrate, Journal of Applied Polymer Science 94 (2004) 2528-2537.

[32] W.-J. Li, J.A. Cooper Jr., R.L. Mauck, R.S. Tuan, Fabrication and characterization of six electrospun poly( $\alpha$-hydroxy ester)-based fibrous scaffolds for tissue engineering applications, Acta Biomaterialia 2 (2006) 377-385.

[33] S. Dash, P.N. Murthy, L. Nath, P. Chowdhury, Kinetic modeling on drug release from controlled drug delivery systems, Acta Poloniae Pharmaceutica - Drug Research 67 (2010) 217-223.

[34] R.W. Korsmeyer, R. Gurny, E. Doelker, P. Buri, N.A. Peppas, Mechanisms of solute release from porous hydrophilic polymers, International Journal of Pharmaceutics 15 (1983) 25-35.

[35] C. Block, M. Furman, Association between intensity of chlorhexidine use and micro-organisms of reduced susceptibility in a hospital environment, Journal of Hospital Infection 51 (2002) 201-206.

[36] L. Grönroos, J. Mättö, M. Saarela, A. Luoma, H. Luoma, H. JousimiesSomer, L. Pyhälä, S. Asikainen, S. Alaluusua, Chlorhexidine susceptibilities of mutans streptococcal serotypes and ribotypes, Antimicrobial Agents and Chemotherapy 39 (1995) 894-898.

[37] C.G. Emilson, Susceptibility of various microorganisms to chlorhexidine, European Journal of Oral Sciences 85 (1977) 255-265.

[38] G. Denton, Chlorhexidine, in: B. SS (Ed.), Disinfection, Sterilization, and Preservation, Lippincott Williams \& Wilkins, Philadelphia, 2001, pp. 321-324.

[39] T.D. Hennessey, Some antibacterial properties of chlorhexidine, Journal of Periodontal Research 8 (1973) 61-67. 